\title{
Editorial
}

\section{Uncertainty Management in Smart Environment}

\author{
Jianbing Ma, ${ }^{1}$ Yong Deng, ${ }^{2}$ Huiyu Zhou, ${ }^{3}$ and Hongchuan $\mathrm{Yu}^{4}$ \\ ${ }^{1}$ School of Computing, Electronics and Mathematics, Coventry University, Coventry CV1 5FB, UK \\ ${ }^{2}$ College of Computer and Information Science, Southwest University, Chongqing 400715, China \\ ${ }^{3}$ School of Electronics, Electrical Engineering and Computer Science, Queen's University Belfast, Belfast BT7 1NN, UK \\ ${ }^{4}$ National Centre for Computer Animation, Bournemouth University, Bournemouth BH12 5BB, UK
}

Correspondence should be addressed to Jianbing Ma; magicmjb@gmail.com

Received 21 November 2016; Accepted 23 November 2016; Published 5 March 2017

Copyright (C) 2017 Jianbing Ma et al. This is an open access article distributed under the Creative Commons Attribution License, which permits unrestricted use, distribution, and reproduction in any medium, provided the original work is properly cited.

The deployment of smart devices has created a body of new application domains: ambient intelligence, Internet of things, industrial control and monitoring systems, assistive living, intelligent surveillance, and so forth. In these applications, quantitative information is usually gathered from sensors, which inevitably contain uncertainty, and possibly inconsistency, due to reasons such as tampered or malfunctioning sensors. In addition, postprocessing of sensor data (e.g., transmission via sensor networks, and video feature analytics) also brings in uncertainty and ambiguity. To utilize the data, uncertainty management approaches have been incorporated in practical applications of smart environments.

The primary purpose of this special issue is to organise a collection of recently developed uncertainty management techniques in the fields of smart environments. Papers selected for this special issue present new findings and insights into this field.

In the paper "An Extended VIKOR Method for Multiple Attribute Decision Analysis with Bidimensional Dual Hesitant Fuzzy Information," M. Xue et al. presented an extension of the fuzzy VIKOR method by introducing a new generalized distance measure between two bidimensional dual hesitant fuzzy sets (BDHF) to solve complex multiple attribute decision analysis problems. The paper also develops a new entropy measure on the BDHF information to fix the extended VIKOR method. The performance of the method is evaluated and analysed in a project aiming at improving people's living quality.

In the paper "An Intelligent Complex Event Processing with $D$ Numbers under Fuzzy Environment," F. Xiao proposed an intelligent complex event processing method with $D$ numbers under fuzzy environment which is based on the Technique for Order Preferences by Similarity to an Ideal Solution (TOPSIS) method. The method fully supports decision making in complex event processing systems.

In the paper "A Modified Model of Failure Mode and Effects Analysis Based on Generalized Evidence Theory," D. Zhou et al. proposed a new generalized evidential failure mode and effects analysis (GEFMEA) model to handle the uncertain risk factor in the conventional FMEA model. In GEFMEA, not only the conventional risk factors, the occurrence, severity, and detectability of the failure mode, but also the other incomplete risk factors as well as the relative importance among all these risk factors are taken into consideration. The efficiency and usefulness of the method are verified in a case study of aircraft turbine rotor blades.

In the paper "Sensor Data Fusion Based on a New Conflict Measure," W. Jiang et al. introduced a modified cosine similarity to measure the similarity between vectors and extended the measure to basic probability assignments (BPAs). The new measure is justified by its nice properties in efficiently measuring the degree of conflict among bodies of evidence.

In the paper "A New Wavelet Threshold Function and Denoising Application," L. Jing-yi et al. proposed basic principles of wavelet threshold denoising and traditional structures threshold functions and presented improved wavelet threshold functions and fixed threshold formulae. Theoretical analysis and experimental results showed that the proposed approach improves the different decomposition scales that 
adopt the same threshold value to deal with the noise problems, also effectively filters the noise in the signals, and improves the signal-to-noise (SNR) and reduces mean square errors (MSE) of output signals. The range of both methods and applications demonstrates the vitality of this research area.

In the paper "Using an Integrated Group Decision Method Based on SVM, TFN-RS-AHP, and TOPSIS-CD for Cloud Service Supplier Selection," L. Li et al. presented how to solve a cloud service supplier selection problem by integrating several uncertainty management techniques. Firstly, a support vector machine (SVM) based classification model is used to reduce the size of the suppliers, and then a fuzzy number rough sets-AHP method is introduced that incorporates experts' knowledge to compute supplier's index values. The index weights are identified by criteria importance from intercriteria correlation and hence the suppliers are assessed by an improved Euclidean distance. The method proves itself in an electric power case study.

We hope that our special issue offers a timely view of the advances in uncertainty management applied in smart environments.

\section{Acknowledgments}

We sincerely express our gratitude to the authors for their contributions and the reviewers for their insightful comments.

Jianbing $M a$ Yong Deng

Huiyu Zhou

Hongchuan $\mathrm{Yu}$ 


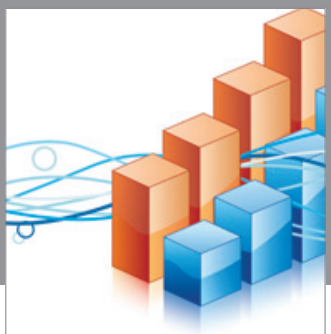

Advances in

Operations Research

vatem alat4

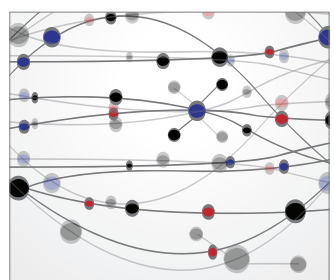

\section{The Scientific} World Journal
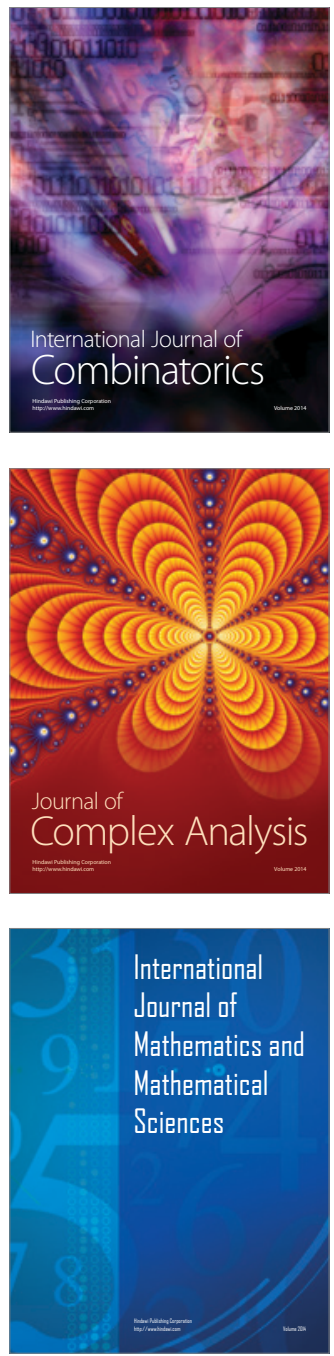
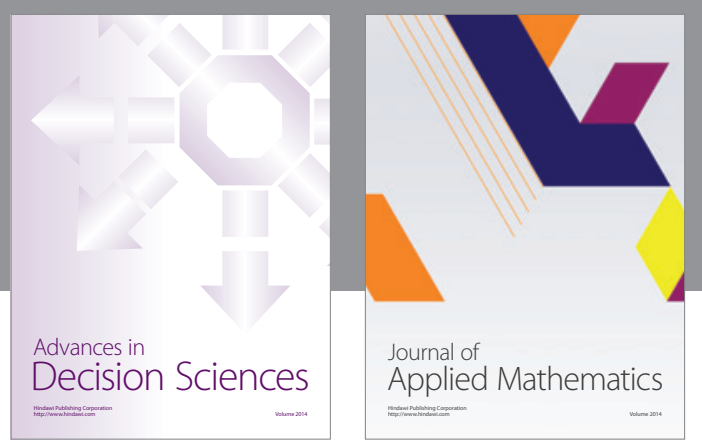

Algebra

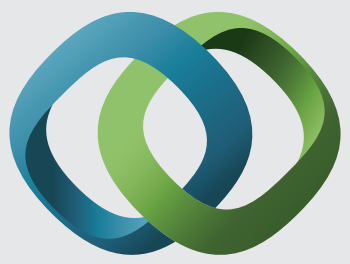

\section{Hindawi}

Submit your manuscripts at

https://www.hindawi.com
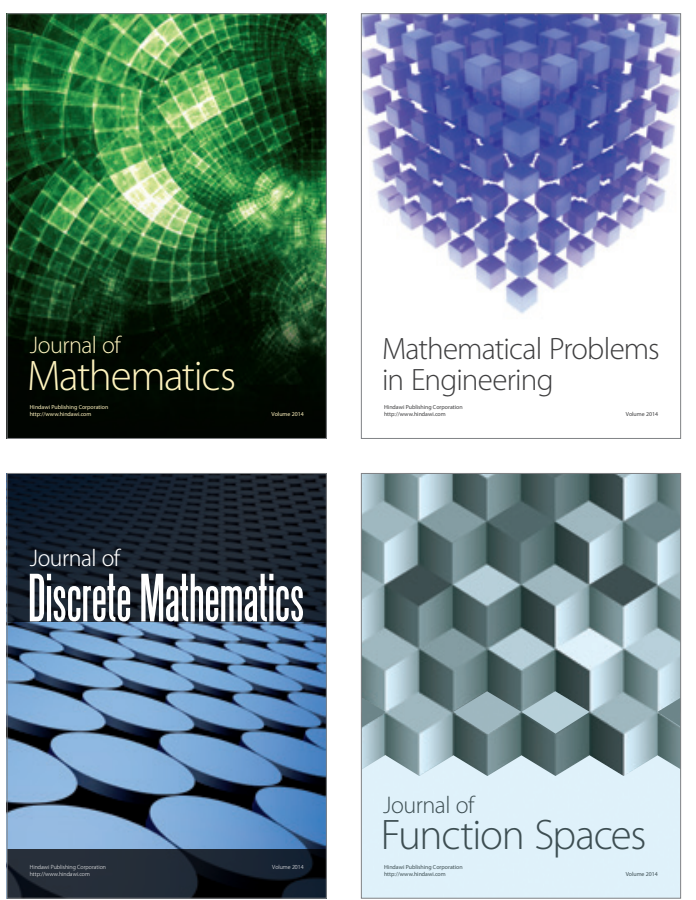

Mathematical Problems in Engineering
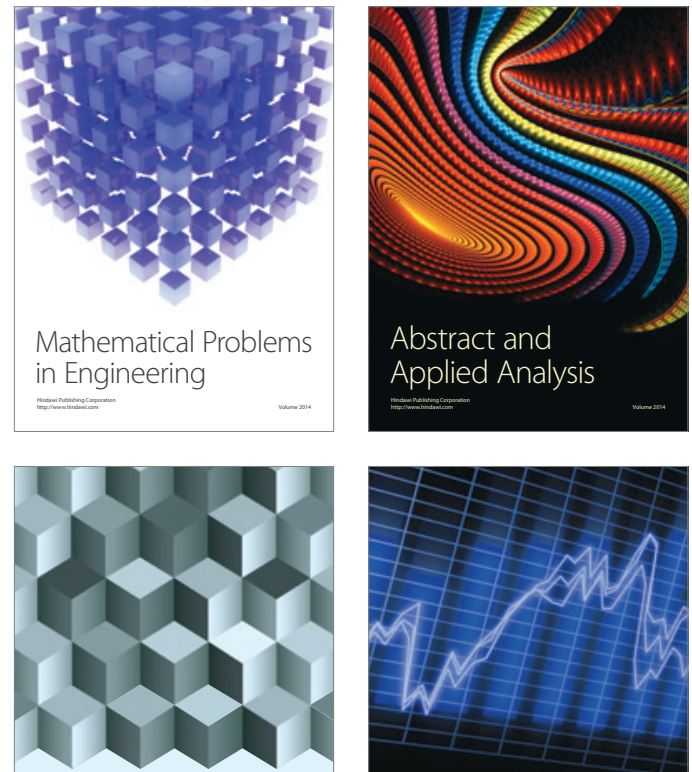

Journal of

Function Spaces

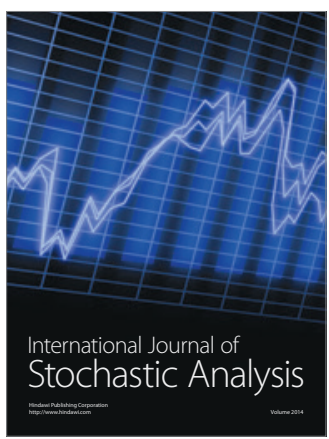

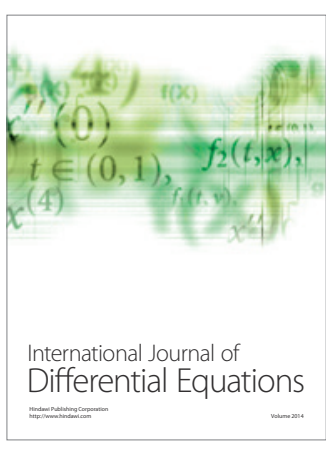
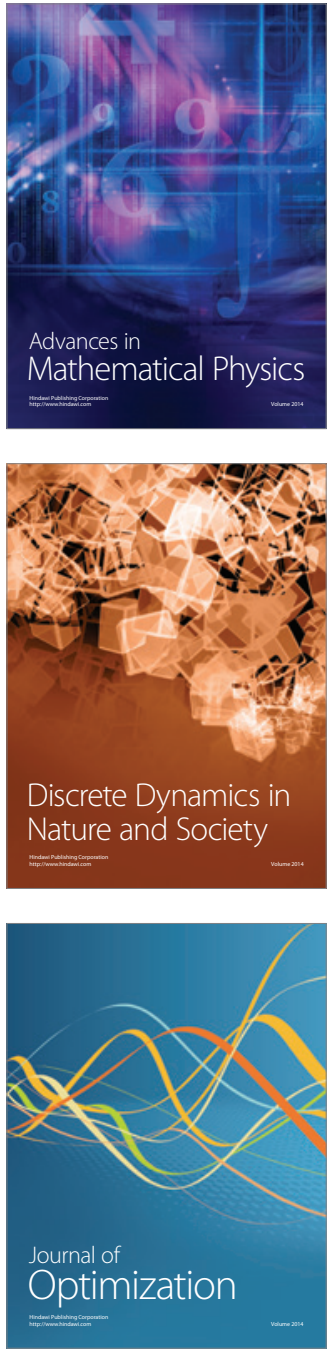